Karl Sperling, Roman M. Marek, Frank Rühli, Nicole Bender, Antoine Flahault und Detlev Ganten

\title{
Ein holistisches Gesundheitskonzept: Die Evolutionäre Medizin
}

\begin{abstract}
Evolutionary Medicine and its Holistic Concept of Health. Recent years have seen tremendous advances in our understanding of biological processes on genomic, cellular, and evolutionary levels. We owe this progress in great part to modern genetic techniques, steady improvements in imaging technology, and ground-breaking molecular tools. These findings not only helped turning Darwin's hypothesis on the origin of species into an exact science, they also require us to integrate the complex interactions between biology, environment, and behavior into our ways of thinking. As a result, a new scientific rationale for a holistic concept of health and disease emerged: Evolutionary Medicine. The holistic approach of Evolutionary Medicine provides a new perspective on human biology: Why are people so frail, why do we get sick? Most importantly, it helps us comprehend how to better preserve health - as opposed to merely focusing on the treatment of diseases. For example, it is the misalignment between our evolutionary 'old' biology and our fast-changing, man-made environment (e.g., urbanization and nutrition with processed food) that helps to comprehend the emergence of civilization diseases.
\end{abstract}

\section{Einleitung}

Big Data, Digitalized Health, Artificial Intelligence, e-Health, Personalized Medicine, Evolutionary Medicine, One Health, Public Health und Global Health sind nur einige der Schlagworte, die einen Aufbruch in eine „Neue Medizin“ verheißen

\footnotetext{
Karl Sperling, Interdisziplinäre Arbeitsgruppe „Zukunft der Medizin: Gesundheit für alle“, Berlin-Brandenburgische Akademie der Wissenschaften; Institut für Medizinische Genetik und Humangenetik, Charité - Universitätsmedizin Berlin, Berlin, Deutschland; Geteilte Erstautorenschaft
}

Roman M. Marek, Interdisziplinäre Arbeitsgruppe „Zukunft der Medizin: Gesundheit für alle“, Berlin-Brandenburgische Akademie der Wissenschaften; Geteilte Erstautorenschaft Frank Rühli, Nicole Bender, Institut für Evolutionäre Medizin, Universität Zürich, Schweiz Antoine Flahault, Institut de santé globale, Université de Genève, Genf, Schweiz Detlev Ganten, Interdisziplinäre Arbeitsgruppe „Zukunft der Medizin: Gesundheit für alle“, Berlin-Brandenburgische Akademie der Wissenschaften; World Health Summit, Charité Universitätsmedizin Berlin, Berlin, Deutschland

Ә OpenAccess. (c) 2021 Karl Sperling, Roman M. Marek, Frank Rühli, Nicole Bender, Antoine Flahault und Detlev Ganten, publiziert von De Gruyter. (cc) BY-NC-SA Dieses Werk ist lizenziert unter einer Creative Commons Namensnennung - Nicht kommerziell - Weitergabe unter gleichen Bedingungen 4.0 International Lizenz. https://doi.org/10.1515/9783110713336-018 
und globale Herausforderungen signalisieren. Tatsächlich führten in den letzten Jahrzehnten verbesserte technische Möglichkeiten, die z. B. die Omics-Technologien (Genomics, Proteomics, Metabolomics etc.) oder Big Data-Analysen bieten, zu bahnbrechenden Erkenntnissen über zelluläre und molekulare, letztlich aber auch die gesamte Tier- und Pflanzenwelt betreffende Zusammenhänge. Folge dieser Forschungsergebnisse war die Einsicht, dass wir alle Teil eines homöostatischen Netzwerkes sind, welches das Ergebnis eines langen evolutionären Prozesses ist, in dem endo- und exogene Faktoren aufs engste zusammenwirken (Ganten et al. 2009). Vor diesem Hintergrund muss das Phänomen „Krankheit“ als Störung eines hochkomplexen homöostatischen Netzwerkes angesehen werden, das in jedem von uns unterschiedlich ausgeprägt ist. Entsprechend variabel sind individuelle Reaktionen auf Veränderungen in diesem reaktiven Netzwerk, entsprechend individuell sollte die medizinische Betreuung sein. Es geht dabei nicht wie in der traditionellen Medizin um die unmittelbaren Krankheitsursachen (Wie kommt es zu einer Erkrankung?), sondern um die grundlegende Ursache, die sich aus dem entwicklungsgeschichtlichen Kontext ergibt (Warum tritt diese Krankheit zu diesem Zeitpunkt bei dieser Person auf?). Diese neue Sichtweise ist die Sichtweise der evolutionären Medizin (Evolutionary Medicine).

Für die gesundheitliche Versorgung zieht die Sichtweise der evolutionären Medizin jedoch noch weitere, tiefergreifende Konsequenzen nach sich, die den Kern der Medizin betreffen: Die Entschlüsselung unserer DNA-Sequenz und die revolutionären neuen molekularen Einsichten in die Biologie und Herkunft des Menschen zwingen Medizin und Wissenschaft geradezu dazu, nicht nur eine immer individuellere, sondern gleichzeitig auch eine holistische Perspektive einzunehmen. Diese ganzheitliche Sichtweise dient 1.) als Grundlage der molekularen Anatomie, Physiologie und Pathologie und ermöglicht dadurch 2.) ein neues Verständnis pathophysiologischer Prozesse; erst durch sie nämlich lassen sich 3.) fachübergreifende Zusammenhänge aufzeigen, die 4.) eine neue Sicht auf Gesundheit und Krankheit eröffnen um 5.) neue Ansätze für Prävention und Therapie zu entwickeln (Brüne \& Schiefenhövel 2019; Buselmaier 2015; Ganten \& Nesse 2012; Gluckman et al. 2016; Nesse \& Williams 1996; Rühli et al. 2016; Stearns \& Koella 2008; Stearns \& Medzhitov 2015; Trevathan et al. 1999). In Forschung, Lehre und Praxis ist eine ganzheitliche Betrachtung deshalb zwar höchst relevant, aber derzeit noch weit entfernt von ihrer Realisierung:

Die praktizierte Medizin beschränkt sich [...] bislang auf die [proximaten] (unmittelbaren) Ursachen einer Krankheit, also auf die physiologischen, anatomischen und heute auch teilweise molekularen bzw. genetischen Voraussetzungen. Der Mensch wird dabei isoliert und nicht als Produkt einer 3 Mrd. Jahre langen Entstehungsgeschichte betrachtet. Die Folge: Grundlegende evolutionsbiologische Ursachen für Gesundheit und Krankheit werden erst gar nicht beachtet und damit nicht tiefgreifend verstanden (Buselmaier 2015, S. VII). 
Nach den epochalen Fortschritten der Medizin im 19. und 20. Jahrhundert schien der Mensch dauerhaft die Oberhand gewonnen zu haben - vor allem im Kampf gegen Infektionskrankheiten. Doch vor allem die sich bei jeder neuen Antibiotikageneration mit geradezu ernüchternder Regelmäßigkeit wiederholende Entwicklung von Resistenzen zwang die wissenschaftliche Forschung dazu, evolutionäre Prozesse stärker zu berücksichtigen. Zugleich scheint sich das Auftreten neuer Pathogene zu häufen - die COVID-19 Pandemie stellt hier nur einen aktuellen, dramatischen Höhepunkt dar, dem weitere folgen werden (Kaufmann 2008).

Bei den damit verbundenen enormen globalen Herausforderungen ist Deutschland zwar in vielen Bereichen der Medizin führend, weist aber gleichzeitig gravierende Defizite auf. So wurde bereits erkannt, dass die IT-Infrastruktur insgesamt und speziell auch im Gesundheitswesen nicht annähernd auf dem Stand ist, der notwendig wäre, um im internationalen Wettbewerb zu bestehen. Zudem gibt es in Deutschland nicht nur gegenüber der Genetik, sondern auch gegenüber der Nutzung von Gesundheitsdaten eine Skepsis, sowohl in der Bevölkerung als auch in Fachkreisen. Dies führt dazu, dass die Erfassung von Daten (z.B. aus Krankenkassendaten und populationsbezogenen Vorsorge- und Früherkennungsuntersuchungen) bewusst nicht digital erfolgt und hierfür datenschutzrechtliche Gründe angeführt bzw. teilweise vorgeschoben werden. So werden große Chancen für die Versorgung vertan und aus unserer Sicht ist es unethisch, vorhandenes Wissen und Möglichkeiten bewusst nicht zum Wohle und für die Gesundheit einzusetzen. Der Schutz persönlicher Daten muss und kann dabei gewährleistet werden.

Ein weiteres, massives Defizit betrifft die Beteiligung am Genomprojekt und den damit einhergehenden technischen und bioinformatischen Entwicklungen sowie den Einsatz der Hochdurchsatzsequenzierung (HDS) in der medizinischen Diagnostik. So sind die Kosten für die Sequenzierung eines Genoms dank der HDS von 100 Mio. US \$ im Jahr 2001 auf ca. 1.000 \$ gesunken, die erforderliche Zeit konnte auf 50 Stunden reduziert werden (Saunders et al. 2012). Heute bereits verfügbare Sequenzierungssysteme werden die Kosten weiter reduzieren. England ist das erste Land weltweit, das 2018 die Sequenzierung des gesamten Genoms bereits in die Routinediagnostik eingeführt hat. Damit kann bei nahezu der Hälfte der Patienten mit Verdacht auf eine seltene genetisch bedingte Entwicklungsstörung eine molekulare Diagnose gestellt und eine oftmals jahrelange diagnostische Odyssee vermieden werden. Eine immer größere Bedeutung spielt die Genomsequenzierung auch für Tumorpatienten: Abgesehen von der Frage einer genetischen Disposition für bestimmte Tumore (Präventionsmedizin) geht es um die genetische Klassifizierung der Tumore im Hinblick auf eine individualisierte Krebstherapie. Wenn man bedenkt, dass die Grundlage der Cancerogenese 
(spontane) Mutationen sind (in Verbindung mit Selektionsprozessen), passt dies ebenfalls in den Rahmen der Evolutionären Medizin. Das Bundesministerium für Gesundheit plant jetzt im Rahmen von genomDE die Genommedizin in die Gesundheitsversorgung zu integrieren. Dies wird nach Ansicht der Bundesregierung

\footnotetext{
zu einem tieferen Verständnis von Krankheitsmechanismen beitragen und neue Wege für die Diagnostik, Prävention und personalisierte Behandlung von Patientinnen und Patienten aufzeigen. Von diesen Entwicklungen werden nach Ansicht der Bundesregierung zunächst insbesondere Patientinnen und Patienten mit seltenen und onkologischen Erkrankungen profitieren (Deutscher Bundestag 2020, S. 2).
}

Es bleibt zu hoffen, dass es gelingt, im Rahmen von genomDE (Deutscher Bundestag 2020) den bestehenden Rückstand aufzuholen. Eine faktenbasierte Analyse des Erreichten, eine wissenschaftlich fundierte Abschätzung des Potentials der Hochdurchsatzmethoden (Farnaes et al. 2018; Gilissen et al. 2014) und der daraus entstehenden Daten sowie die öffentliche Kommunikation erscheinen aufgrund der Aktualität, der hierfür einzusetzenden Ressourcen und einer Koordinierung der Aktivitäten in Deutschland mehr als dringlich, worauf immer wieder hingewiesen wurde (Gießelmann 2019; Propping et al. 2010; Robinson et al. 2008; Ropers 2018; Ropers et al. 2015). Dabei sind es von allen Omics-Technologien vor allem die Genomics, d.h. die Genomforschung, die am meisten zum Paradigmenwechsel hin zur Evolutionären Medizin beigetragen haben (Ganten \& Nesse 2012): Im Zentrum steht das Gen (Ganten et al. 2009).

\section{Genetik und Genomik als Grundlagen der Evolutionären Medizin}

Bis in die Mitte des vergangenen Jahrhunderts bildete Virchows Cellularpathologie die fachübergreifende Grundlage für weite Bereiche der Medizin (Virchow 1858). Heute nimmt die molekulare Pathologie diese Position ein, welche sich auf ein tiefer liegendes Erklärungsprinzip stützt, nämlich auf Veränderungen im Erbgut. Damit ist der direkte Anschluss an die Evolutionstheorie und die Evolutionäre Medizin gegeben (Sperling 2020). Die Begründung hierfür resultiert aus der 1885 von August Weismann aufgestellten Theorie von der Kontinuität der Keimbahn (Weismann 1885). Danach wurde die Weitergabe der genetischen Information seit Anbeginn des Lebens niemals unterbrochen, das Erbgut eines jeden Individuums repräsentiert also die im Laufe der Stammesgeschichte bis zu ihm angesammelten Veränderungen. Dank des größten biomedizinischen Forschungsvorhabens unserer Zeit, des Humangenomprojekts (Sperling 2000), ist die vollständige Abfolge 


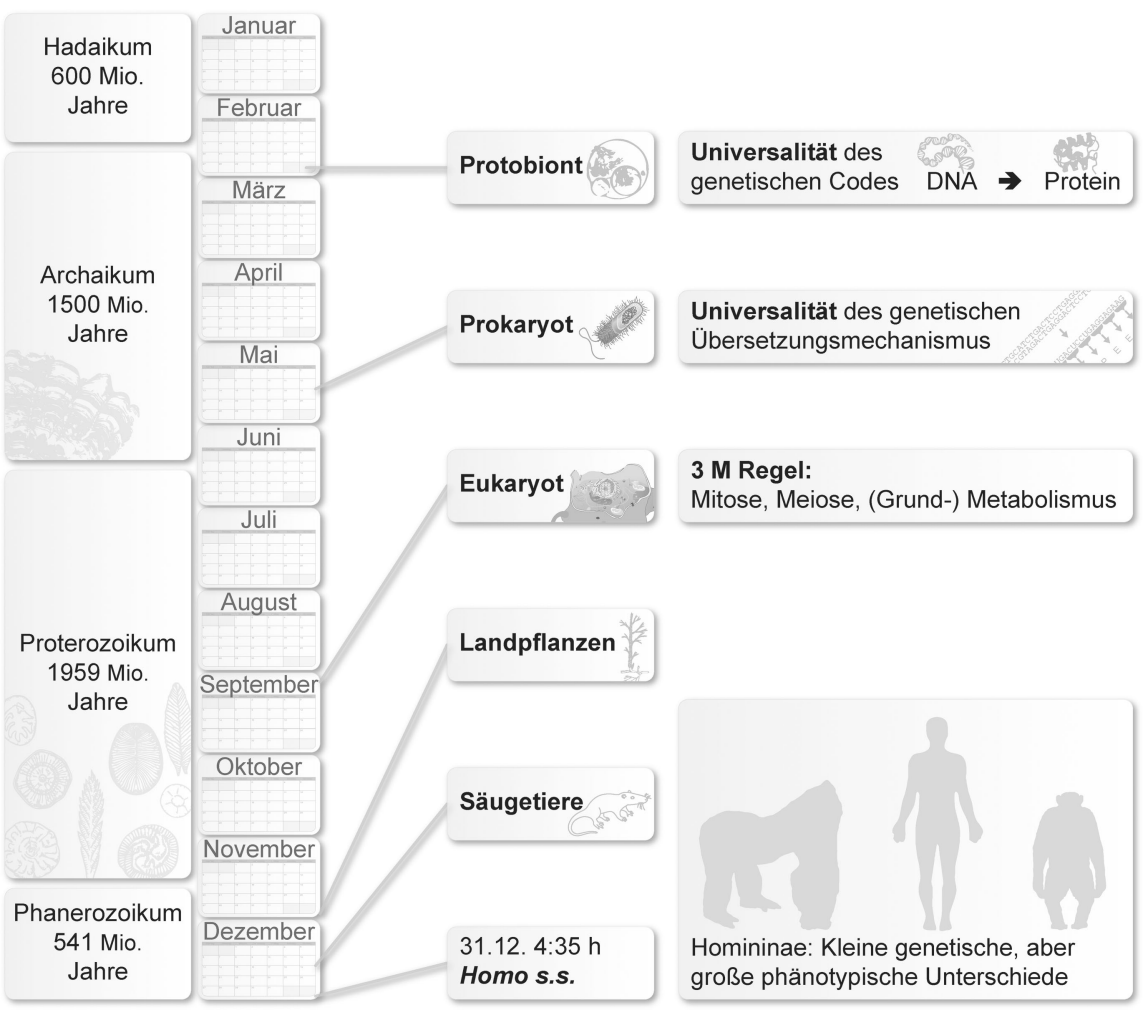

Abb. 1: Die Evolution des Lebens auf ein Jahr komprimiert, Grafik: Roman Marek, Vergleich der Größe von Gorilla (Gorilla gorilla), Schimpanse (Pan troglodytes) und Menschen nach: https://commons.wikimedia.org/wiki/File:Comparison_of_size_of_western_gorilla_(Gorilla_go rilla),_chimpanzee_(Pan_troglodytes)_and_human.svg, CC-BY-SA-4.0

der einzelnen Bausteine des menschlichen Erbgutes inzwischen bekannt. Die Funktion der einzelnen Bausteine erschließt sich nicht zuletzt aus dem evolutionären Kontext, wobei etwas vereinfacht gilt: Je wichtiger ein Abschnitt in der DNA ist, desto stärker ist er in der Evolution konserviert.

Komprimiert man die Evolution des Lebens von der Entstehung der Erde an auf ein Jahr (Abb. 1) (Sperling 2020), entstanden die ersten Lebensformen (Protobiont) Ende Februar. Die „Universalität“ des genetischen Codes ist noch heute Beleg aus dieser Zeit. Mitte Mai traten die ersten prokaryotischen Zellen (Bakterien) auf. Dass Bakterien heute z. B. menschliches Insulin bilden können, ist auf die Übereinstimmung im genetischen Übersetzungsapparat zurückzuführen. Die Eukaryoten, zu denen alle Pflanzen und Tiere einschließlich des Menschen zählen, treten erstmals Anfang September auf. Ihre zelluläre Ultrastruktur stimmt 
noch weitgehend überein, ebenso der Ablauf von Mitose, Meiose und des (Grund-)Metabolismus (3 M-Regel). Die damals entstandenen Gene blieben bis zum heutigen Zeitpunkt weitgehend erhalten. Dies zeigt sich z. B. an den monogen bedingten Krankheiten beim Menschen. In den überwiegenden Fällen sind so genannte Haushaltsgene betroffen, die sich bereits bei einzelligen Pflanzen und Pilzen finden. Nur fünf Prozent dieser Gene treten erst bei Vielzellern auf (Lee et al. 2020). Insgesamt wurden viele der Gene, die auch beim Menschen den Zellzyklus oder die Proteinsekretion steuern, zuerst bei Einzellern identifiziert. Es überrascht daher nicht, dass grundlegende physiologische Eigenschaften an Modellorganismen entdeckt und danach auf den Menschen übertragen wurden, wie umgekehrt auch die Analyse monogen bedingter Krankheiten des Menschen zum Verständnis derart fundamentaler Vorgänge beigetragen hat. Im Prinzip ist daher jeder molekulare Genetiker in der Medizin zugleich auch Evolutionsforscher, denn er trägt dazu bei, evolutionär konservierte biologische Prozesse $z u$ entschlüsseln.

Die Entwicklung ist aber kein genetisch fest vorgegebener, sondern ein epigenetischer Prozess. Danach geht das Individuum aus der Befruchtung einer Eizelle durch ein Spermium hervor. In den jeweiligen Zellkernen findet sich die genetische Information in Form der DNA-Doppelhelix und beide Eltern tragen nahezu gleich viel zum Vererbungsgeschehen bei. Da praktisch alle Körperzellen durch Zellteilung aus der befruchteten Eizelle hervorgehen, enthalten sie im Prinzip auch sämtliche Erbanlagen. Dass sich die verschiedenen Gewebe unterscheiden, beruht letztlich darauf, dass jeweils nur bestimmte Gene aktiviert sind. Die Embryonalentwicklung ist daher ein epigenetischer Prozess, der auf dem noch weitgehend unverstandenen Wechselspiel vieler tausend Erbanlagen mit exo- und endogenen Umweltfaktoren beruht und auch den Zufall miteinschließt.

Die Konsequenzen aus dieser scheinbar trivialen Feststellung werden häufig übersehen: Gene für Intelligenz oder bestimmte charakterliche Veranlagung kann es nicht geben, da es sich hierbei ja erst um Ergebnisse sehr komplexer Entwicklungsprozesse handelt. Die Gleichsetzung eines Gens mit einem sichtbaren Merkmal (Phän) ist daher unzulässig. Zugleich gilt es zu bedenken, dass genetische Information nur über die Keimzellen weitergegeben wird. Daher können Eigenschaften, die während des individuellen Lebens erworben wurden, nicht vererbt werden. Damit steht jedoch nicht im Widerspruch, dass bestimmte epigenetische Veränderungen sehr wohl an die Nachkommen weitergegeben werden können. So hat als einer der ersten Günter Dörner von der Charité - Universitätsmedizin Berlin darauf hingewiesen, dass unphysiologische Dosen von Hormonen, Neurotransmittern und Zytokinen, die während der Embryonalentwicklung einwirken, später zu lebenslangen Störungen des Stoffwechsels, der Fortpflanzung und der Immunantwort führen können (Plagemann 2014). 
Überraschend war auch, dass die protein-kodierenden Gene weniger als 2\% des menschlichen Genoms ausmachen, während etwa die Hälfte aus repetitiven Elementen besteht. So haben vor etwa 25 Millionen Jahren Retroviren das Genom unserer Primatenvorfahren besiedelt und sich durch Transposition darin vermehrt (Cordaux \& Batzer 2009). Ebenso war unerwartet, dass auf DNA-Niveau der Schimpanse mehr Übereinstimmung mit dem Menschen als mit dem Gorilla aufweist. Es kommt daher zu dem Paradoxon, dass die phänotypischen Unterschiede zwischen Mensch und Schimpanse eine Zuordnung zu verschiedenen Familien rechtfertigten, während die genetischen Unterschiede nicht größer als die zwischen Arten einer Gattung sind. Die Frage stellt sich daher, wie können in evolutionär so kurzer Zeit bei einer derart hohen genetischen Übereinstimmung so weitreichende Veränderungen eingetreten sein, wie der aufrechten Gang und die enorme Zunahme des Neocortex?

Für derart weitreichende Veränderungen kommen grundsätzlich Mutationen in Strukturgenen und/oder in regulatorischen Elementen infrage. Da die meisten Strukturgene aber in vielfältige, verschiedene Entwicklungsprozesse einbezogen sind, würden sich hier Veränderungen auf verschiedene Prozesse gleichzeitig auswirken, sie wären deshalb prinzipiell eher nachteilig. Aus diesem Grund genießen Strukturgene einen besonderen Schutz, sie sind evolutionär konserviert. Regulatorische Elemente hingegen wirken sich deutlich spezifischer aus, bei ihnen herrscht deshalb mehr Flexibilität. Wesentliche morphologisch-anatomische Unterschiede zwischen Mensch und Menschenaffen sind daher auf veränderte Entwicklungsabläufe zurückzuführen, die entscheidend von Änderungen in der Regulation der Genaktivität abhängen dürften. Dafür sprechen evolutionäre Veränderungen in der Anzahl spezifischer regulatorischer DNAs, aber auch die zahlenmäßigen Veränderungen solcher Gene, die im Neocortex aktiv sind (Sperling 2015).

Überraschende Erkenntnisse erbrachte auch der DNA-Vergleich heute lebender Menschen: So sind die genetischen Unterschiede innerhalb der einzelnen Ethnien deutlich größer als die Unterschiede zwischen ihnen. Dies deutet darauf hin, dass sich die einzelnen Ethnien erst seit relativ kurzer Zeit getrennt entwickelt haben. Zugleich zeigte sich, dass die genetischen Unterschiede zwischen verschiedenen Bevölkerungsgruppen in Afrika deutlich größer sind als z.B. zwischen Europäern und Asiaten. Die Erklärung hierfür ist, dass der Ursprung der heutigen Menschen in Afrika liegt und die Auswanderung erst vor weniger als 100.000 Jahren erfolgte. Der vor 2 Millionen Jahren aufgetretene Frühmensch, †Homo erectus, wanderte ebenfalls aus Afrika aus, es handelt sich jedoch bei diesen Populationen um ausgestorbene Seitenzweige und nicht um direkte Vorfahren des modernen Menschen. 
Nicht alle Seitenzweige verschwanden spurlos (Fu et al. 2015; Pääbo 2014; Slon et al. 2018; Warren 2018). So konnte gezeigt werden, dass es nach der Auswanderung des Homo sapiens sapiens aus Afrika unter anderem zu einer Verbindung mit dem Neandertaler (†Homo sapiens neanderthalensis) gekommen ist, dessen Reste von Erbgut sich bei Eurasiern, nicht jedoch bei Afrikanern nachweisen lassen. Es handelt sich dabei vor allem um solche Gene, die Haut und Haar betreffen und die eine Anpassung an die kälteren klimatischen Bedingungen begünstigt haben dürften.

Eine Besonderheit des Menschen ist, dass er als einziger Primat das schützende Haarkleid (Fell) verlor, wodurch die Haut dem intensiven Sonnenlicht direkt ausgesetzt war. Die dunkle Hautfarbe der Afrikaner ist ein essentieller Schutz gegen das UV-Licht, gegen dessen erbgutschädigende Wirkung und die Zerstörung der lebenswichtigen Folsäure. Die Lichtstärke war aber noch ausreichend, um in den Zellen der Haut das Vitamin D (D3) zu bilden. Mit der Auswanderung des Menschen aus Afrika in die lichtärmeren Breiten der Erde und deren erheblichen jahreszeitlichen Schwankungen in der Lichtstärke änderten sich diese Verhältnisse grundlegend. Die hellere Hautfarbe ist ein Ausdruck davon, ebenso auch die Korrelation der Hautfarbe mit der jahreszeitlichen Lichtstärke. Die Pigmentierung zählt damit zu dem variabelsten Merkmal des Menschen überhaupt (Jablonski \& Chaplin 2010). Diese Anpassung an die veränderten Umweltbedingungen erfolgte über hunderte von Generationen. Heute können sich Auswanderer in wenigen Stunden in geographischen Regionen ansiedeln, denen sie evolutionär nicht angepasst sind. Dies hat weitreichende gesundheitliche Auswirkungen, sowohl für hellhäutige Menschen in den Tropen als auch für dunkelhäutige in den nordischen Breiten, wenn nicht entsprechende Gegenmaßnahmen vorgenommen werden.

Bei Hellhäutigen kann dies zu einem Mangel an Folsäure führen, der bei Föten ein erhöhtes Risiko zur Bildung von Neuralrohrdefekten (Spina bifida) zur Folge haben kann (Parra 2007), außerdem entwickelt sich leichter Hautkrebs (Williams et al. 2013). Dunkelhäutige haben hingegen eher unter einem Mangel an Vitamin D zu leiden, der das Risiko für bestimmte Krebsarten (Brust, Prostata, Dickdarm) erhöht (Jablonski \& Chaplin 2010), und außerdem zu mangelhafter Knochenbildung (bis hin zu Rachitis) führen, aber auch das Immunsystem (mit einem erhöhten Risiko für Autoimmunkrankheiten) und die Zellproliferation beeinträchtigen kann.

Aus genetischer Sicht spricht alles dafür, dass die Kulturfähigkeit des Menschen schon in der Altsteinzeit vor der Auswanderung aus Afrika gegeben war. Die entscheidende Voraussetzung hierfür war die enorme Zunahme des Gehirns, genauer der Großhirnrinde, deren Volumen größer ist als das gesamte Gehirn eines Schimpansen. Die Größenzunahme des Gehirns ist beim Menschen aber nicht wie 
bei den anderen Primaten mit der Geburt abgeschlossen. Das menschliche Neugeborene ist daher unreifer und hilfsbedürftiger als das aller anderen Säugetiere und in besonderem Maße auf Fürsorge und Schutz durch das soziale Umfeld angewiesen. Es spricht vieles dafür, dass die Zunahme der kognitiven Fähigkeiten in der menschlichen Stammesgeschichte eng mit dem Zusammenleben in komplexen Sozialverbänden zusammenhängt, sprachliche Verständigung dürfte hier eine entscheidende Rolle gespielt haben. Die anatomischen und genetischen Voraussetzungen zur Ausbildung der Sprache besaß vermutlich bereits der gemeinsame Vorfahre von Mensch und Neandertaler (Sperling 2015).

Das Sprachvermögen ist zugleich Grundlage des begrifflichen Denkens und damit der Entstehung menschlicher Kulturfähigkeit. Das heißt, die entscheidende Umwelt des Menschen ist der Mensch. Das unbewusste Erlernen der Sprache ist jedoch an eine bestimmte Entwicklungsphase gebunden. Vereinfacht gilt, dass in dieser sensitiven Periode genetisch festgelegte Entwicklungsabläufe in Verbindung mit bestimmten sensorischen Reizen zur Bildung neuer neuronaler Verbindungen (Synapsen) führen. Bleiben die exogenen Stimuli aus, kommt es auch zum Absterben von Nervenzellen und die entsprechenden Lernvorgänge können später nur schwer oder gar nicht nachgeholt werden. Die Entwicklung der Kulturfähigkeit in der Ontogenese beruht daher auf einer phylogenetisch bedingten genetischen Veranlagung, die in einer bestimmten Entwicklungsphase durch die jeweilige Umwelt ihre Ausprägung erfährt.

Im Verband mit Sesshaftigkeit, Ackerbau und Viehzucht markiert die neolithische Revolution vor ca. 10.000 Jahren einen weiteren Meilenstein der kulturellen Evolution. Als Folge davon haben die Infektionskrankheiten zugenommen und als starke Selektionsfaktoren gewirkt, was seinen Niederschlag im Erbgut gefunden hat. Hinzu kamen Veränderungen in Anpassung an die neuen Ernährungsbedingungen. Mit der Entwicklung der ersten Schrift in Mesopotamien und Ägypten gegen Ende des 4. Jahrtausend v. u. Z. wird die Sprache dann auf ein dauerhaftes Fundament gestellt. Mit der Renaissance und der Aufklärung kam es in Verbindung mit der Industrialisierung $\mathrm{zu}$ einem beispiellosen naturwissenschaftlichen und technologischen Fortschritt, wobei sich mit Erfindung der Buchdruckkunst eine ganz neue Dimension der Verbreitung des Geschriebenen eröffnete. Heute wird dieser Fortschritt extrem gesteigert durch die digitale Revolution, die intensive internationale Kooperation und entscheidend befördert durch eine einheitliche Wissenschaftssprache: Englisch als lingua franca. Die extreme Bevölkerungszunahme in den letzten 200 Jahren ist Ausdruck dieser kulturellen und nicht einer darwinschen Evolution (Sperling 2015). Der Kulturfähigkeit des Menschen liegt danach eine genetisch bedingte Anpassungsfähigkeit zugrunde, wobei die jeweilige Kultur manifester Ausdruck der Angepasstheit 
ist. So gesehen, „ist die Kultur die wahre Natur des Menschen, seine spezifische Weise der Anpassung an die Welt“ (Markl 1998, S. 6).

Das, was den Menschen auszeichnet, ist sein Verstand. Unser Gehirn ist aber nicht entstanden, um die Wahrheit zu erkennen, sondern um das Überleben im Mesokosmos, den mittleren Dimensionen, $\mathrm{zu}$ sichern. Allerdings hat die Evolution uns Fähigkeiten verliehen, die weit über das hinausgehen, wofür sie ursprünglich selektioniert wurden, weshalb wir den Mesokosmos auch überschreiten können (siehe Quantenphysik). Evolutionär von zentraler Bedeutung war zudem, aus der Vielzahl der Sinneseindrücke jene zu extrahieren, die kausal in Beziehung zueinanderstehen. Diese Anpassung ist jedoch nicht ideal und mit Fehlleistungen verbunden. So geht damit die Veranlagung einher, kausale Beziehungen zu Dingen herzustellen, die nichts miteinander zu tun haben (z. B. das menschliche Schicksal mit der Konstellation der Gestirne). Da unsere Denkweise das jüngste Ergebnis der Evolution ist, ist sie auch durch die darwinsche Selektion am wenigsten erprobt (Vollmer 2016). „Reinen Unsinn zu glauben, ist ein Privileg des Menschen“, sagt Konrad Lorenz. Entsprechend stellt Hubert Markl fest: „Der Mensch ist vor allem „Kausalitätssuchmaschine mit der Neigung, Glaubensvorstellungen über schier alles, was er erlebt, zu entwickeln“, er ist ein „Ursachensucher und Ursachen(er)finder“ (Markl 2008). Es fällt nicht schwer, Beispiele hierfür auch aus der Medizin anzuführen. Die Evolutionäre Erkenntnistheorie macht hier verständlich, welchen unbewussten Zwängen unsere kognitiven Fähigkeiten unterliegen.

Stark vereinfacht kann man sagen, dass die frühen Entwicklungsphasen eines Menschen stärker genetisch bestimmt sind als die späteren und dass eine Störung des genetischen Netzwerkes als Folge einer Genmutation daher generell schwerwiegendere klinische Konsequenzen hat und sich häufig als monogen bedingte Krankheit äußert. Mehr als $80 \%$ davon manifestieren sich bis zur Pubertät. Danach überwiegen die komplexen oder multifaktoriell bedingten Krankheiten. Dazu zählen die Herz-Kreislauferkrankungen, Diabetes oder Psychosen, von denen jeweils einige Prozent der Bevölkerung betroffen sind. In diesen Fällen liegen häufig genetische Polymorphismen oder Varianten vor, die im Zusammenspiel mit anderen Faktoren genetischer und umweltbedingter Art zu der jeweiligen Krankheit führen. Die Polymorphismen zählen zur normalen Variabilität des Erbgutes, denen unter anderen Umweltverhältnissen durchaus ein Vorteil zukommen konnte. So muss man immer bedenken, dass der Mensch die weitaus längste Zeit seiner Stammesgeschichte nomadisch als Jäger und Sammler lebte und seine genetische Ausstattung daher auch primär diesen Lebensumständen angepasst ist. Damals gab es kein Überangebot an Nahrung, keinen Bewegungsmangel und keine Computerspiele. Evolutionär sind wir nur einge- 
schränkt an die globalisierte Welt angepasst, was auch die Zunahme psychischer Störungen (mit) erklären könnte.

So, wie sich die Menschen aufgrund ihrer Erbanlagen äußerlich unterscheiden, sind sie auch verschieden im Hinblick auf ihre normalen physiologischen Eigenschaften. Damit verbunden ist die individuell unterschiedliche Reaktion auf die Einnahme bestimmter Medikamente, die unterschiedliche Anfälligkeit gegenüber Infektionserregern, aber auch die unterschiedliche Disposition für HerzKreislauf-Erkrankungen. In vielen Fällen dürfte diese genetisch bedingte Variabilität Ausdruck einer Anpassung an bestimmte (frühere) Umweltbedingungen sein. Dieses auf Garrod zurückgehende Konzept von der biochemischen (heute würden wir sagen: genetischen) Individualität (Garrod 1909) begreift Krankheit nicht einfach als Gegensatz zu Gesundheit, sondern als Störung eines homöostatischen Netzwerkes, welches das Ergebnis eines langen evolutionären Prozesses ist. Entsprechend variabel ist die individuelle Reaktion auf genetisch bedingte Veränderungen in diesem Netzwerk, entsprechend individuell sollte die medizinische Betreuung sein. Im Prinzip bedeutet dies eine Abkehr von einer überwiegend typologisch ausgerichteten hin zu einer stärker individuell orientierten Medizin.

Bei allen Fragen an die zukünftige Genomforschung bleiben folgende wesentliche Erkenntnisse bestehen: 1.) Alle Lebewesen sind viel enger miteinander verwandt als zuvor gedacht; 2.) die meisten zellulären Bausteine finden sich über alle Artgrenzen hinweg; 3.) die evolutionäre Entwicklung wesentlicher Baupläne lässt sich konkret über den zeitlichen Verlauf nachweisen; und 4.) bestätigt sich die Hypothese der Evolution als großes Experiment zufälliger, nicht zielgerichteter Entwicklung, deren Ergebnisse immer abhängig von der jeweiligen Umgebung und Geschichte zu bewerten sind (Sperling 2001). Hinzu kamen noch überraschende Erkenntnisse, z. B. der Nachweis des Austausches von Erbmaterial über alle Artgrenzen hinweg. So stammt auch beim menschlichen Erbgut ein beachtlicher Anteil ursprünglich von Viren und Bakterien ab (Crisp et al. 2015).

Auf die Bedeutung von Neumutationen für das Evolutionsgeschehen wurde hier immer wieder hingewiesen. Entscheidend dabei ist jedoch die Mutationsrate, wie eine einfache Überlegung zeigt: Angesichts der Größe des menschlichen Erbguts treten allein aufgrund physiko-chemischer Gegebenheiten spontan pro Tag und Zellkern Zehntausende DNA-Einzelstrangbrüche und Tausende Basenverluste auf. Ohne wirkungsvolle DNA-Reparaturmechanismen gäbe es praktisch keine intakten Keimzellen. Die DNA ist daher das einzige Makromolekül der Zelle, das bei einer Schädigung repariert und nicht durch ein neues Molekül ersetzt wird. Dies ist eine Voraussetzung für das Leben schlechthin. Die Reparaturprozesse gehören daher zu den evolutiv besonders alten Errungenschaften. Dies er- 
klärt auch, dass bestimmte Prozesse, die beispielhaft an Bakterien und Hefen erschlossen wurden, auch für menschliche Zellen gelten (Sperling 2016).

Allerdings treten pro Generation immer noch etwa 80 Basenaustausche als Neumutationen auf, die meisten davon paternalen Ursprungs. Mit jedem väterlichen Lebensjahr nimmt deren Zahl um 1-2 zu (Goldmann et al. 2016; Veltman \& Brunner 2012). Diese spontanen Mutationen können erhebliche klinische Relevanz haben, wenn sie die Funktion von Strukturgenen beeinträchtigen und z. B. zu mentaler Retardierung führen (Gilissen et al. 2014). Die Mutationsrate von Genund Chromosomenmutationen kann zudem durch Umweltnoxen, wie ionisierende Strahlen, erhöht werden (Holtgrewe et al. 2018).

\section{Was unterscheidet die Evolutionäre Medizin vom traditionellen Verständnis von Gesundheit?}

Wie bereits erwähnt, geht es bei der traditionellen Medizin um die unmittelbaren (proximaten) Krankheitsursachen, die durch eine Untersuchung der anatomischen und physiologischen Gegebenheiten aufgedeckt werden. Im Vordergrund steht die Frage: Wie kommt es zu einer Erkrankung (Gegenstand ist der Phänotyp)? Die Evolutionäre Medizin hingegen sieht den Organismus als das Produkt einer 3 Milliarden Jahre langen Geschichte an und fragt, warum tritt diese Krankheit bei dieser Person zu diesem Zeitpunkt auf (Gegenstand ist der Genotyp). Beide Sichtweisen ergänzen sich. Die Antworten der evolutionären Medizin reichen jedoch tiefer, da es hier um die grundlegenden (ultimaten) Krankheitsursachen geht.

Die Evolutionäre Medizin sieht unseren Körper folglich nicht als ausgereifte und durchdachte Maschine, die nach einem Plan entworfen wurde. Stattdessen ist der derzeitige Stand als das Ergebnis eines Prozesses zu sehen, der sich über Milliarden von Jahren erstreckt hat, und der das Ergebnis unzähliger winziger Veränderungen ist, die unter den jeweiligen Umweltbedingungen kleine Verbesserungen darstellten. Vieles wurde im Laufe der Zeit ,mitgeschleppt‘, auch wenn sich die Umwelt- oder Lebensbedingungen drastisch geändert hatten. Keineswegs wurde immer die optimale Lösung gefunden, vielmehr wurde etwas bereits Vorhandenes angepasst. Das Auge z. B. gilt als „unvollkommenes Konstruktionsmerkmal“ des menschlichen Körpers aufgrund evolutionärer Beschränkungen: Die Photorezeptoren (Stäbchen und Zapfen) sind dem Licht abgewandt, im Gegensatz zum Auge des Tintenfischs. Die Konstruktion der Wirbelsäule zur Erlangung des aufrechten Ganges belegt, welche Vorteile damit für die Mensch- 
werdung verbunden waren, aber auch welche Nachteile hinsichtlich der Anfälligkeit für bestimmte Krankheiten damit in Kauf genommen werden mussten. So unangenehme Beschwerden wie Schmerzen und Fieber, Angst und Depressionen sind als Abwehr- und Schutzmechanismen entstanden. Darüber hinaus haben andere Lebewesen ihre Spuren in unserem Erbgut hinterlassen. Man könnte unseren Körper somit als verstetigtes Provisorium aus lauter zusammengewürfelten Notlösungen verstehen. Das Paradox ist nun, dass dieser Zustand unseren Körper einerseits so anfällig für Krankheiten macht, ihn gleichzeitig aber auch überraschend widerstandsfähig und anpassungsfähig hält (Nesse \& Stearns 2008). Die Evolutionäre Medizin sucht hier nach den Kompromissen im Aufbau, die entwicklungsgeschichtlich eingegangen werden mussten und bietet mehr Hinweise auf die Prävention von krankhaften Entwicklungen.

Ein weiterer wichtiger Aspekt der Evolutionäre Medizin ist die Erkenntnis, dass unsere Entwicklungsgeschichte eben nicht auf ein langes und gesundes Leben ausgerichtet war, sondern auf reproduktive Fitness (Jasienska et al. 2017). Wenn eine Genvariante einen positiven Effekt in der Jugend mit sich bringt, dann setzt sie sich durch, selbst wenn sie im Alter nachteilige Wirkung entfalten wird (antagonistische Pleiotropie, s.u.): Jugend sticht Alter (Ganten et al. 2009). Falls Krankheiten also eine genetische Komponente beinhalten, so muss man sich immer fragen, weshalb sich diese Gene entwicklungsgeschichtlich erhalten haben. Entscheidend ist hier auch das Konzept des Mismatch: Genvarianten, die uns ursprünglich beim Überleben geholfen haben, stellen nun eine Bedrohung dar. Nicht, weil sie ,schlecht‘ sind, sondern weil sich die Umweltbedingungen drastisch und schnell verändert haben. Hier fragt die Evolutionäre Medizin verstärkt nach der Rolle von Umwelteinflüssen auf krankhafte Veränderungen und nach der Rolle von Genen unter früheren Lebensbedingungen oder in anderen Lebewesen. Gerade im Hinblick auf die Ernährung und Prävention spielen diese Überlegungen eine große Rolle.

Auch im Hinblick auf die Symptome fordert die Evolutionäre Medizin eine erweiterte Perspektive ein. Die Symptome einer Krankheit werden nicht einfach bekämpft; zunächst gilt es zu klären, welche direkt durch die Krankheit verursacht werden und welche auf die Abwehrmaßnahmen unseres Köpers zurückzuführen sind. Dies kann von Erreger zu Erreger unterschiedlich sein, im jeweiligen Fall muss genau unterschieden werden, welche Reaktion unseres Körpers uns, und welche dem Erreger zugutekommt (Buselmaier 2015, S. 51-52). Das Augenmerk gilt der Ursache einer Krankheit, nicht ihren Symptomen.

Die Evolutionäre Medizin ist von Natur aus eine stark interdisziplinär ausgerichtete Wissenschaft, sie vereint Erkenntnisse aus den verschiedensten Fachbereichen, von der Archäologie über die Humangenetik bis zur Paläobiologie. Der Hintergrund ist, dass Erkenntnisse aus grauer Vorzeit oder der Stoffwechsel eines 
anaerob auf dem Boden der Ozeane lebenden Bakteriums für die Behandlung heutiger Patienten große Relevanz haben können. Ebenso verschwinden die Grenzen zwischen den verschiedenen Arten (One Health), denn die Evolutionäre Medizin hat alle Lebewesen im Blick - um uns zu verstehen und um uns selbst zu helfen. Aktuelle Herausforderungen wie der Klimawandel, Pandemien oder Antibiotikaresistenzen lassen sich nur in einer globalen Perspektive lösen, dies lenkt den Fokus noch mehr auf die Gesundheit des gesamten Planeten (Planetary Health) - selbst wenn wir nur rein egoistisch an unsere eigene Gesundheit denken wollen (Beaglehole \& Yach 2003; Chopra et al. 2002; Kaufmann 2008; The Lancet 2017a). Damit erstreckt sich die ganzheitliche Perspektive der Evolutionären Medizin auf alle Lebewesen, auf den gesamten Planeten, und auf unsere gemeinsame Vergangenheit seit Anbeginn des Lebens. Letztliches Ziel ist dabei, Krankheiten nicht nur immer besser zu verstehen und zu behandeln, sondern ihre Entstehung zu verhindern. Gerade hier liegt die große Chance der Evolutionären Medizin (Nesse \& Stearns 2008).

Das aus der evolutionären Betrachtungsweise resultierende neue Verständnis von Krankheit kann z. B. an folgenden Beispielen illustriert werden:

- Angeborene Fehlbildungen: Die Ontogenese der Wirbeltiere beruht auf der Musterbildung sog. Entwicklungsfelder, bei denen es sich „um die fundamentalen Einheiten der Entwicklung und der Evolution“ handelt (Opitz \& Rauch 1999). Daran beteiligten Genfamilien finden sich bereits bei Invertebraten, Untersuchungen haben hier zu grundlegenden Einsichten in die Entstehung von Fehlbildungen beim Menschen geführt (Kantaputra et al. 2010). Durch die evolutionäre Analyse struktureller Varianten konnten zudem am Beispiel seltener Entwicklungsstörungen grundlegende Einsichten in die Regulation der Genaktivität gewonnen werden (Spielmann et al. 2012; Spielmann et al. 2018). Allgemein gilt, dass angeborene Fehlbildungen sog. Entwicklungsfelddefekte darstellen, sie haben daher auch eine evolutionäre Entsprechung. In einigen Fällen handelt es sich um die Persistenz eines Embryonalstadiums, wie Neuralrohrdefekte (Spina bifida), das Vorhandensein eines Schwanzes oder Schwimmhäute zwischen den Fingern. Andere dieser Fehlbildungen betreffen Strukturen, die aus evolutionärer Sicht noch relativ neu, und damit fehleranfälliger sind. So ist z. B. bei den meisten Reptilien, also unseren evolutionären Vorfahren, eine einfache Gaumenspalte normal, ebenso wie zwei unvollständig getrennte Herzkammern. Beim Menschen aber zählen Gaumenspalten und Ventrikelseptumdefekte zu den häufigsten Fehlbildungen.

- Die Mukoviszidose ist bei uns mit 1:2500 unter Neugeborenen eine der häufigsten autosomal-rezessiven Stoffwechselerkrankungen. Jeder 25. ist heterozygoter Genträger. Mehr als 1000 Mutationen sind bekannt, jedoch weisen 
in Deutschland mehr als $70 \%$ eine Mutation auf $(\Delta \mathrm{F} 508)$, bei der heterozygote Träger in den Alveolen der Lunge weniger Cl-Ionenkanäle aufweisen (Bertranpetit \& Calafell 1996). Die Cl-Ionenkanäle stellen die Eintrittspforte für Typhusbakterien dar. Im Mausmodell konnte gezeigt werden, dass die heterozygoten Träger der Mutation weniger anfällig gegenüber Typhusbakterien waren (Pier et al.1998). Es wird daher vermutet, dass dies auch bei Menschen so ist und sich daher diese Mutation so verbreiten konnte.

- Das Gen für das Enzym Glukose-6-Phosphat Dehydrogenase (G6PD) liegt auf dem X-Chromosom. Die Mutation zu G6PD-Defizienz ist in Gebieten mit Malaria weit verbreitet und betrifft $\sim 400$ Millionen Personen weltweit. Es ist damit der häufigste Enzym,defekt' (Howes et al. 2012). Ebenso wie der Träger des Gens für Sichelzellanämie weisen Personen mit G6PD-Defizienz einen Vorteil gegenüber der Malaria auf. Sie sind ansonsten gesund, entwickeln jedoch nach Einnahme bestimmter Medikamente (Antimalaria Mittel, Sulfonamide, ASS/Aspirin, Nitrofurantoin, Quinidine, Purin Glycoside) eine Hämolyse. Dieses ist nur ein Beispiel für die individuell unterschiedliche Reaktion auf die Einnahme von Medikamenten. So wird derzeit (Juli 2021) für 83 Wirkstoffen eine genetische Diagnostik vor der Behandlung verlangt und für weitere 9 Wirkstoffe empfohlen (Die forschenden Pharma-Unternehmen 2021). Derartige pharmakogenetische Reaktionen können auch deutliche ethnische Unterschiede aufweisen. Das bekannteste Beispiel dafür ist die Cytochrom-P450-abhängige Monooxygenase CYP2D6, die bei der Verstoffwechselung von nahezu 25\% der gebräuchlichsten Arzneistoffe, insbesondere Psychopharmaka vom Typ der SSRI, Betablocker und Schmerztherapeutika, beteiligt ist. Dabei wird zwischen langsamen, schnellen und sehr schnellen Metabolisierern unterschieden. In Deutschland finden sich ca. 3\% sehr schnelle Metabolisierer, im Mittelmeerraum und in Teilen Afrikas hingegen bis zu 10 oder 20\% (Kirchheiner 2003).

- Alterserscheinungen: Eine der wichtigsten Erklärungen ist hier das Konzept der antagonistischen Pleiotropie (Williams 1957). Nach dieser Theorie gibt es bestimmte Genvarianten, die im jugendlichen Alter, d.h. der reproduktiven Phase, vorteilhaft sind und weitergegeben werden (s.o.). Im höheren Alter wirken diese sich dann aber negativ aus, nur unterliegen sie dann nicht mehr der darwinschen Selektion, da die reproduktive Phase bereits abgeschlossen ist. Ein Beispiel dafür könnte eine Mutation im Faktor-V-Gen der Blutgerinnung sein, die sich bei 5\% der kaukasischen Bevölkerung findet und die das Thromboserisiko bei älteren Menschen erhöht. Als Erklärung für die erhöhte Häufigkeit wird angenommen, dass im jugendlichen Alter eine raschere Blutgerinnung bei der Menstruation oder nach Verletzungen von Vorteil ist, jedoch mit einem Nachteil (Thrombosebildung) in höherem Alter einhergeht 
(Lindqvist \& Dahlback 2008). Es gibt experimentelle Belege und Untersuchungen an Modellorganismen, die die Theorie der antagonistischen Pleiotropie stützen, ebenso eine Reihe von Beobachtungen am Menschen, bei denen es sich jedoch überwiegend um Korrelationen und keine direkten kausalen Beweise handelt. Insgesamt dürfte die antagonistische Pleiotropie den wichtigsten evolutionären Mechanismen darstellen, um das Altern von Organismen und ihren natürlichen Tod zu erklären (Austad \& Hoffman 2018; Barbi et al. 2018a, 2018b; Beltrán-Sánchez et al. 2018; Burger et al. 2012; Byars \& Voskarides 2019; Dong et al. 2016).

- Bei den meisten Tieren ist die durchschnittliche Lebenserwartung mit dem Ende der Fortpflanzungsfähigkeit erreicht. Eine der wenigen Ausnahmen ist der Mensch. Die Lebensdauer der Frauen reicht weit über die Menopause hinaus. Als eine Erklärung wird der Großmutter-Effekt angenommen. Als Folge der enormen Zunahme des Gehirns ist das menschliche Neugeborene nicht nur hilfsbedürftiger als das aller anderen Säugetiere, sondern der Geburtsvorgang für die Mutter auch besonders riskant. Die Großmutter kann sie unterstützen, und im Falle des Todes sogar an die Stelle der Kindsmutter treten. Hinweise auf diesen Großmuttereffekt haben sich in ursprünglichen (pre-modernen) Bevölkerungen in Finnland und Kanada nachweisen lassen (Hawkes 2004; Lahdenperä et al. 2004). Auch die unterschiedlichen Sterberaten zwischen den Geschlechtern beim Menschen können evolutionsbiologisch begründet werden (Kruger \& Nesse 2006).

Folgt man dem Denkansatz der evolutionären Medizin, so muss man die bisherige phänomenologische Einteilung der Krankheiten im Sinne der Osler'schen (1892) Vorgehensweise nach Manifestationsalter, Geschlecht bzw. betroffenem Organsystem durch eine zunehmend ätiologisch orientierte Klassifikation ergänzen, die zugleich eine ganzheitlichere Betrachtungsweise des Krankheitsgeschehens bedingt. Auf diese Weise wird der Zersplitterung in der Medizin entgegengewirkt und es werden fachübergreifend Zusammenhänge aufgezeigt, die zugleich Grundlage einer molekularen Anatomie und Physiologie sind, dem Verständnis pathophysiologischer Prozesse dienen und bestimmte ökologische Zusammenhänge verständlich machen.

\section{Kritik an der Evolutionären Medizin}

Da heute der naturwissenschaftlichen Medizin ein naturalistisches Weltbild zugrunde liegt, braucht in diesem Kontext nicht auf religiös motivierte Argumente gegen die Evolutionstheorie eingegangen $\mathrm{zu}$ werden. Wie bei allen naturwissen- 
schaftlich-atheistischen Betrachtungsweisen muss aber auch bei der Evolutionären Medizin darauf geachtet werden, dass der Aspekt der ,Spiritualität‘ hinsichtlich der psychischen Gesundheit und des Wertes des Lebens nicht vernachlässigt wird. An dieser Stelle sei warnend auf den beispiellosen medizinischen Missbrauch hingewiesen, der gestützt auf - meist falsch verstandene oder missinterpretierte - evolutionsbiologische bzw. sogenannte ,sozialdarwinistische' Vorstellungen von Eugenik und Rasse begangen wurde. Von diesen auf Halbwissen beruhenden Fehlinterpretationen der Evolutionstheorie distanziert sich die Evolutionäre Medizin aufs Schärfste.

Selbstverständlich unterlag auch die Evolutionstheorie selbst einer Entwicklung, sie wird auch weiterhin erweitert, und noch immer gibt es offene Fragen, die innerhalb der Wissenschaft umstritten sind und offen thematisiert werden (Kutschera \& Niklas 2004). Unstrittig ist, dass es sich bei der Evolutionstheorie um eine historische Disziplin handelt, die sich anfangs nur auf Beobachtungen, Annahmen und deren Interpretation stützte. Inzwischen aber konnten unzählige experimentelle Untersuchungen an Modellorganismen zum Evolutionsgeschehen und die gefundenen mathematischen Gesetzmäßigkeiten jeglichen Zweifel am Faktum der Evolution ausräumen. Wie bei allen über Modelle oder Tierexperimente gewonnenen Erkenntnissen ist bei der Übertragung auf den Menschen Vorsicht geboten. Doch in letzter Zeit hat die molekulare Analyse tausender Genome die Evolutionstheorie, gerade auch im Hinblick auf ihre medizinische Relevanz, wesentlich erweitert (Stearns 2017). Im Nachhinein - d. h. vor dem Hintergrund unseres heutigen Wissens - mögen viele der über Jahrhunderte gewonnen Erkenntnisse natürlich trivial oder selbstverständlich erscheinen.

Ein valider Kritikpunkt hingegen ist, dass die Sichtweise der Evolutionären Medizin offensichtlich begrenzt ist, da die natürliche Selektion primär auf den Reproduktionserfolg gerichtet ist und nicht auf ein langes Leben in Gesundheit. Sie steht daher unter dem Generalverdacht einer geringen praktischen Relevanz (Uncommon Descent 2018) und wird oft als medizinische Grundlagenwissenschaft (wie etwa die Biologie) angesehen. Angesichts des übervollen Lehrplans im Medizinstudium ist daher nur noch wenig Platz für die Evolutionäre Medizin. Schließlich geht es für die betroffenen Patienten in der Therapie um die proximaten Ursachen, und nicht um die ultimaten. Über die Epigenetik kommt auch der anderen Vererbungslehre - nach der sich Lebewesen aktiv an veränderte Umweltbedingungen anpassen und dies dann an ihre Nachkommen weitergeben - eine medizinisch immer wichtigere Rolle zu. Selbstverständlich hat der Lebensstil Auswirkungen auf die Genaktivität der Keimzellen und embryonalen Stammzellen und darüber auf die Gesundheit von Kindern und Enkelkindern ohne dass dabei die Gensequenz selbst verändert wird. Auch diese Sichtweise ist 
daher Gegenstand der Evolutionären Medizin. Für sie trifft der bekannte Aphorismus zu „Es gibt nichts Praktischeres als eine gute Theorie“.

\section{Zusammenfassung und Empfehlungen}

Gesundheit ist ein Menschenrecht und Gesundheit hat nicht nur für die einzelne Person sondern für die Gesellschaft insgesamt einen besonderen Stellenwert. Es zeigte sich aber, dass Gesundheit mehr ist als Medizin. Gesundheit umfasst die gesamte Biologie des Menschen einschließlich unseres evolutionären Ursprungs, unserer Umwelt (z. B. Urbanisierung, Verschmutzung, Konflikte, Klimawandel), und unser Verhalten (Kultur, Ernährung, Bewegung, Bildung). Zudem wird Gesundheit maßgeblich beeinflusst von den vielfachen Einflüssen der sich verändernden Umwelt auf unsere Biologie (Planetary Health). Die enge Einbindung des Menschen in die Tier- und Pflanzenwelt sowie die der Mikroorganismen, z. B. durch von Tieren übertragene Infektionskrankheiten (wie AIDS, die Spanische Grippe, Trichinen, Vogelgrippe, Ebola, COVID-19) haben zu dem umfassenden Konzept One Health geführt. Für die Medizin der Zukunft bedeutet dies, dass sie über die klassischen Bereiche Diagnostik, Therapie, Prävention hinaus Gesundheit aus einer holistischen Perspektive betrachten muss.

In Forschung, Lehre und Praxis steht immer noch die Krankheit selbst im Zentrum, eine holistische Perspektive ist nicht etabliert. Hier wäre dringend geboten, das Konzept der Evolutionären Medizin mit seinen präventiven und evolutionären Aspekten im Schulunterricht und medizinischen Curriculum zu verankern. Bei allen vielversprechenden Ansätzen übernimmt die Wissenschaft noch zu wenig Verantwortung im Hinblick auf Globale Gesundheit (Ganten et al. 2018), sie ist auf den Gebieten Public Health und Global Health schlecht aufgestellt: Globale Gefahren durch Seuchenausbrüche in Entwicklungsländern werden vernachlässigt, dabei erfordern Pandemien globale Herangehensweisen (Mobilität, Antibiotika-Resistenzen). Gerade auf diesem Gebiet ergeben sich über die Sichtweise der Evolutionären Medizin neue und vielversprechende Ansatzpunkte (Kruger 2011; Omenn 2009; The Lancet 2017b), besonders im Hinblick auf eine genauere Precision Public Health, die mehr Rücksicht nimmt auf regionale und populationsspezifische Unterschiede und Bedürfnisse (James et al. 2019; Sheath et al. 2020). Es ist ein Missverständnis, dass die Evolutionäre Medizin nur bei somatischen Krankheiten zur Anwendung kommen kann. Gerade auf dem Gebiet psychischer Erkrankungen verspricht ein Perspektivwechsel neue Erkenntnisse und Therapiemöglichkeiten (Nesse 2019). Störungen wie Depressionen, Essstörungen oder Suchtkrankheiten liegen Mismatch-Komponenten zugrunde, meist im Hinblick auf ein fehlendes oder falsches soziales Umfeld in unseren modernen 
Gesellschaften, oder es gibt einen Mismatch zwischen unserer evolutionären psychischen Ausstattung und unserem modernen Angebot an Nahrungs- und Genussmitteln. Bei Phänomenen wie Schizophrenie oder Autismus ließe sich aus evolutionärer Sicht hinterfragen, ob es sich überhaupt um ,Krankheiten' handelt, oder ob die Verhaltensweisen aus evolutionärer Sicht unter anderen Umgebungen von Vorteil waren. Vor diesem Hintergrund erscheint unverständlich, dass es im Gegensatz zu anderen europäischen Ländern in Deutschland noch kein Institut für Evolutionäre Medizin gibt.

Die Perspektive der Evolutionären Medizin führt unausweichlich dazu, dass sich die Grenzen zwischen präventiven, diagnostischen und therapeutischen Eingriffen verschieben werden. Hier stellen sich neue ethische Fragen: Was muss oder darf therapiert werden? Neue und verbesserte Diagnose- und Therapiemöglichkeiten im Bereich der Genomik und Präzisionsmedizin stellen zudem eine finanzielle Herausforderung dar, außerdem sind ihre psychischen Auswirkungen auf die Patienten und deren Angehörigen noch unklar. In Bezug auf diese Punkte besteht dringender Forschungsbedarf. Kritisch zu sehen ist auch, dass Gesundheit zunehmend als individuelle Aufgabe verhandelt wird (Verantwortung für die eigene Gesundheit, Lebensstile), damit geht eine gesellschaftliche Entsolidarisierung einher. Dabei sollte allen bewusst sein, dass wir als Gesellschaft nur solidarisch überleben können, dies gilt insbesondere im Hinblick auf die Erreichung der 17 Nachhaltigkeitsziele der Vereinten Nationen (SDGs). Diese geben bereits eine holistische Sicht für die weltweite Verbesserung der Gesundheit vor, leider aber sind die 17 SDGs in Deutschland noch immer nicht in einem ausreichenden Maß implementiert. Die SDGs können wir aber nur erreichen, wenn wir die Perspektive der Evolutionären Medizin einnehmen (Ganten et al. 2018).

\section{Literatur}

Austad, Steven N./Hoffman, Jessica M. (2018): „s Antagonistic Pleiotropy Ubiquitous in Aging Biology?“. In: Evolution, Medicine, and Public Health 2018(1), S. 287-294.

Barbi, Elisabetta/Lagona, Francesco/Marsili, Marco et al. (2018a): „The Plateau of Human Mortality: Demography of Longevity Pioneers“. In: Science 360(6396), S. 1459-1461.

Barbi, Elisabetta/Lagona, Francesco/Marsili, Marco et al. (2018b): „Response to Comment on ,ThePlateau of Human Mortality: Demography of Longevity Pioneers““. In: Science 362(6412), S. eaav3229.

Beaglehole, R./Yach, D. (2003): „Globalisation and the Prevention and Control of Non-communicable Disease: The Neglected Chronic Diseases of Adults“. In: The Lancet 362(9387), S. 903-908.

Beltrán-Sánchez, H./Austad, S. N./Finch, C. E. (2018): „Comment on ,The Plateau of Human Mortality: Demography of Longevity Pioneers““. In: Science 361(6409), S. eaav1200. 
Bertranpetit, Jaume/Calafell, Francesc (1996): „Genetic and Geographical Variability in Cystic Fibrosis: Evolutionary Considerations“. In: Ciba Foundation Symposium 197, S. 97-118.

Brüne, Martin/Schiefenhövel, Wulf (2019): Oxford Handbook of Evolutionary Medicine. Oxford: Oxford University Press.

Burger, O./Baudisch, A./Vaupel, J. W. (2012): „Human Mortality Improvement in Evolutionary Context“. In: Proceedings of the National Academy of Sciences 109(44), S. 18210-18214.

Buselmaier, Werner (2015): Evolutionäre Medizin: Eine Einführung für Mediziner und Biologen. Wiesbaden: Springer Fachmedien.

Byars, Sean G./Voskarides, Konstantinos (2019): „Genes that Improved Fitness also Cost Modern Humans: Evidence for Genes with Antagonistic Effects on Longevity and Disease“. In: Evolution, Medicine, and Public Health 2019(1), S. 4-6.

Chopra, M./Galbraith, S./Darnton-Hill, I. (2002): „A Global Response to a Global Problem: The Epidemic of Overnutrition“. In: Bull World Health Organ 80(12), S. 952-958.

Cordaux, Richard/Batzer, Mark A. (2009): „The Impact of Retrotransposons on Human Genome Evolution“. In: Nature Reviews Genetics 10(10), S. 691-703.

Crisp, Alastair/Boschetti, Chiara/Perry, Malcolm et al. (2015): „Expression of Multiple Horizontally Acquired Genes Is a Hallmark of Both Vertebrate and Invertebrate Genomes“. In: Genome Biology 16(1), S. 50.

Deutscher Bundestag (2020): Drucksache 19/21111. Antwort der Bundesregierung auf die Kleine Anfrage der Abgeordneten Katrin Helling-Plahr, Michael Theurer, Grigorios Aggelidis, weiterer Abgeordneter und der Fraktion der FDP - Drucksache 19/20508 - Deutschlands Beitritt zur ,1+ Million Genomes'-Initiative. Berlin: Deutscher Bundestag, https://dip21. bundestag.de/dip21/btd/19/211/1921111.pdf, besucht am 14.07.2020.

Die forschenden Pharma-Unternehmen (2021): „In Deutschland zugelassene Arzneimittel für die Personalisierte Medizin“. https://www.vfa.de/de/arzneimittel-forschung/ datenbanken-zu-arzneimitteln/individualisierte-medizin.html, besucht am 5.7.2021.

Dong, Xiao/Milholland, Brandon/Vijg, Jan (2016): „Evidence for a Limit to Human Lifespan“. In: Nature 538(7624), S. 257-259.

Farnaes, Lauge/Hildreth, Amber/Sweeney, Nathaly M. et al. (2018): „Rapid Whole-genome Sequencing Decreases Infant Morbidity and Cost of Hospitalization“. In: npj Genomic Medicine 3(1), S. 10.

Fu, Qiaomei/Hajdinjak, Mateja/Moldovan, Oana Teodora et al. (2015): „An Early Modern Human from Romania with a Recent Neanderthal Ancestor“. In: Nature 524(7564), S. $216-219$.

Ganten, Detlev/Nesse, Randolph (2012): „The Evolution of Evolutionary Molecular Medicine: Genomics Are Transforming Evolutionary Biology into a Science with New Importance for Modern Medicine“. In: Journal of Molecular Medicine 90(5), S. 467-470.

Ganten, Detlev/Silva, João Gabriel/Regateiro, Fernando et al. (2018): „Science Has to Take Responsibility. 10 Years World Health Summit - The Road to Better Health for All“. In: Front Public Health 6, S. 314.

Ganten, Detlev/Spahl, Thilo/Deichmann, Thomas (2009): Die Steinzeit steckt uns in den Knochen: Gesundheit als Erbe der Evolution. München: Piper.

Garrod, Archibald E. (1909): Inborn Errors of Metabolism. London: Henry Frowde and Hodder \& Stoughton.

Gießelmann, Kathrin (2019): „Genomsequenzierung: Deutschland steht im Abseits“. In: Deutsches Ärzteblatt International 116(25), S. A-1215/B-1004/C-1992. 
Gilissen, Christian/Hehir-Kwa, Jayne Y./Thung, Djie Tjwan et al. (2014): „Genome Sequencing Identifies Major Causes of Severe Intellectual Disability“. In: Nature 511(7509),

S. $344-347$.

Gluckman, Peter/Beedle, Alan/Hanson, Mark (2016): Principles of Evolutionary Medicine (2 Aufl.). Oxford: Oxford University Press.

Goldmann, Jakob M./Wong, Wendy S. W./Pinelli, Michele et al. (2016):

„Parent-of-origin-specific Signatures of de novo Mutations“. In: Nature Genetics 48(8),

S. $935-939$.

Hawkes, Kristen (2004): „The Grandmother Effect“. In: Nature 428(6979), S. 128-129.

Holtgrewe, Manuel/Knaus, Alexej/Hildebrand, Gabriele et al. (2018): „Multisite de novo Mutations in Human Offspring after Paternal Exposure to lonizing Radiation“. In: Scientific Reports 8(1), S. 14611.

Howes, Rosalind E./Piel, Frédéric B./Patil, Anand P. et al. (2012): „G6PD Deficiency Prevalence and Estimates of Affected Populations in Malaria Endemic Countries: A Geostatistical Model-Based Map“. In: PLOS Medicine 9(11), S. e1001339.

Jablonski, N. G./Chaplin, G. (2010): „Human Skin Pigmentation as an Adaptation to UV Radiation“. In: Proceedings of the National Academy of Sciences 107(Supplement_2), S. $8962-8968$.

James, W. P. T./Johnson, R. J./Speakman, J. R. et al. (2019): „Nutrition and Its Role in Human Evolution“. In: Journal of Internal Medicine 285(5), S. 533-549.

Jasienska, Grazyna/Bribiescas, Richard G./Furberg, Anne-Sofie et al. (2017): „Human Reproduction and Health: An Evolutionary Perspective“. In: The Lancet 390(10093), S. $510-520$.

Kantaputra, Piranit N./Klopocki, Eva/Hennig, Bianca P. et al. (2010): „Mesomelic Dysplasia Kantaputra Type Is Associated with Duplications of the HOXD Locus on Chromosome 2q“. In: European Journal of Human Genetics 18(12), S. 1310-1314.

Kaufmann, Stefan H.E. (2008): Wächst die Seuchengefahr? Globale Epidemien und Armut: Strategien zur Seucheneindämmung in einer vernetzten Welt. Frankfurt am Main: Fischer. Kirchheiner, Julia (2003): „Arzneitherapieempfehlungen auf pharmakogenetischer Basis“. [Habilitationsschrift, Charité - Universitätsmedizin Berlin]. Berlin. https://edoc.hu-berlin. de/bitstream/handle/18452/14589/Kirchheiner.pdf?sequence=1.

Kruger, Daniel J. (2011): „Evolutionary Theory in Public Health and the Public Health of Evolutionary Theory“. In: Futures 43(8), S. 762-770.

Kruger, Daniel J./Nesse, Randolph M. (2006): „An Evolutionary Life-history Framework for Understanding Sex Differences in Human Mortality Rates“. In: Human Nature 17(1), S. $74-97$.

Kutschera, Ulrich/Niklas, KarlJ (2004): „The Modern Theory of Biological Evolution: An Expanded Synthesis“. In: Naturwissenschaften 91(6), S. 255-276.

Lahdenperä, Mirkka/Lummaa, Virpi/Helle, Samuli et al. (2004): „Fitness Benefits of Prolonged Post-reproductive Lifespan in Women“. In: Nature 428(6979), S. 178-181.

Lee, Chelsea E./Singleton, Kaela S./Wallin, Melissa et al. (2020): „Rare Genetic Diseases: Nature's Experiments on Human Development“. In: iScience 23(5), S. 101123.

Lindqvist, Pelle/Dahlback, Bjorn (2008): „Carriership of Factor V Leiden and Evolutionary Selection Advantage“. In: Current Medicinal Chemistry 15(15), S. 1541-1544.

Markl, Hubert (1998): Homo sapiens. Zur fortwirkenden Naturgeschichte des Menschen. Münster: Rhema. 
Markl, Hubert (2008): „Der Mensch - das sich selbst erfindende Wesen“. In: Detlev Ganten/Volker Gerhardt/Jan-Christoph Heilinger/Julian Nida-Rümelin (Hrsg.): Was ist der Mensch? Berlin \& New York: De Gruyter, S. 183.

Nesse, Randolph M. (2019): Good Reasons for Bad Feelings: Insights from the Frontier of Evolutionary Psychiatry. New York, NY: Dutton.

Nesse, Randolph M./Stearns, Stephen C. (2008): „The Great Opportunity: Evolutionary Applications to Medicine and Public Health“. In: Evol Appl 1(1), S. 28-48.

Nesse, Randolph M./Williams, G.C. (1996): Why We Get Sick: The New Science of Darwinian Medicine. New York, NY: Vintage Books.

Omenn, G. S. (2009): „Evolution and Public Health“. In: Proceedings of the National Academy of Sciences 107(Suppl. 1), S. 1702-1709.

Opitz, John M./Rauch, Anita (1999): „Von der befruchteten Eizelle zum Menschen: genetische Defekte als Schlüssel zum Verständnis der menschlichen Ontogenese“. In: Detlev Ganten (Hrsg.): Unsere Welten der Information - Gene, Neurone, Qubits \& Co. Stuttgart: Wissenschaftliche Verlagsgesellschaft, S. 237-254.

Pääbo, Svante (2014): Neanderthal Man: In Search of Lost Genomes. New York, NY: Basic Books.

Parra, Esteban J. (2007): „Human Pigmentation Variation: Evolution, Genetic Basis, and Implications for Public Health“. In: American Journal of Physical Anthropology 134(S45), S. 85-105.

Pier, Gerald B./Grout, Martha/Zaidi, Tanweer et al. (1998): „Salmonella typhi Uses CFTR to Enter Intestinal Epithelial Cells“. In: Nature 393(6680), S. 79-82.

Plagemann, Andreas (2014): „Perinatale Programmierung, neuro-endokrine Epigenomik und präventive Medizin - Das Konzept der Vegetativen Prägung“. In: Nova Acta Leopoldina NF 120, S. $197-225$.

Propping, Peter/Bartram, Claus R./Brandis, Matthias et al. (2010): Prädiktive genetische Diagnostik als Instrument der Krankheitsprävention. Halle an der Saale: Nationale Akademie der Wissenschaften Leopoldina, Deutsche Akademie der Technikwissenschaften, Berlin-Brandenburgische Akademie der Wissenschaften, https:// www.leopoldina.org/uploads/tx_leopublication/201011_natEmpf_praedikative-DE.pdf, besucht am 10.11.2010.

Robinson, Peter N./Köhler, Sebastian/Bauer, Sebastian et al. (2008): „The Human Phenotype Ontology: A Tool for Annotating and Analyzing Human Hereditary Disease“. In: The American Journal of Human Genetics 83(5), S. 610-615.

Ropers, Hans-Hilger (2018): Medizinische Genomsequenzierung: Warum Deutschland nicht länger abseits stehen darf. Berlin: Konrad-Adenauer-Stiftung, https://www.kas.de/ documents/252038/3346186/Analysen+\&+Argumente+324+-+Medizinische +Genomsequenzierung.pdf/b24bfa86-b10a-04d5-2870-843e3f219d79?version=1.1, besucht am 11.11.2020.

Ropers, Hans-Hilger/Diekämper, Julia/Hümpel, Anja (2015): „Themenbereich Gendiagnostik: Hochdurchsatz-Sequenzierung - eine Chance für die genetische Krankenversorgung in Deutschland“. In: Bernd Müller-Röber/Nediljko Budisa/Julia Diekämper/Silke Domasch/Boris Fehse/Jürgen Hampel/Ferdinand Hucho/Anja Hümpel/Kristian Köchy/Lilian Marx-Stölting/Jens Reich/Hans-Jörg Rheinberger/Hans-Hilger Ropers/Jochen Taupitz/Jörn Walter/Martin Zenke (Hrsg.): Dritter Gentechnologiebericht. Analyse einer Hochtechnologie. Baden-Baden: Nomos, Bd. 32, S. 91-147. 
Rühli, Frank/van Schaik, Katherine/Henneberg, Maciej (2016): „Evolutionary Medicine: The Ongoing Evolution of Human Physiology and Metabolism“. In: Physiology 31(6), S. $392-397$.

Saunders, C. J./Miller, N. A./Soden, S. E. et al. (2012): „Rapid Whole-Genome Sequencing for Genetic Disease Diagnosis in Neonatal Intensive Care Units“. In: Science Translational Medicine 4(154), S. 154 ra135.

Sheath, Danny J./Castañeda, Rafael Ruiz de/Bempong, Nefti-Eboni et al. (2020): „Precision Global Health: A Roadmap for Augmented Action“. In: Journal of Public Health and Emergency; Vol 4 (March 2020): Journal of Public Health and Emergency 4(5), S. 1-12.

Slon, Viviane/Mafessoni, Fabrizio/Vernot, Benjamin et al. (2018): „The Genome of the Offspring of a Neanderthal Mother and a Denisovan Father“. In: Nature 561(7721), S. $113-116$.

Sperling, Karl (2000): „Das Humangenomprojekt: Medizin im Licht der Evolution“. In: Deutsche medizinische Wochenschrift 125, S. A15-A20.

Sperling, Karl (2001): „,Altes“ Genom und moderne Umwelt - Lehren aus der Wissenschaft und Problem der Medizin“. In: Karl-Friedrich Wessel/Kurt S. Zänker/Günter Dörner (Hrsg.): Genom und Umwelt. Berliner Studien zur Wissenschaftsphilosophie und Humanontogenetik. Bielefeld: Kleine, Bd. 19, S. 31-47.

Sperling, Karl (2015): „Die Natur-Kultur-Grenze im Licht des Humangenomprojekts“. In: Medizinische Genetik 27(1), S. 7-18.

Sperling, Karl (2016): „Das Humangenomprojekt und die Identifizierung von Krankheitsgenen: DNA-Reparaturdefekte als Modell“. In: Walter Sudhaus (Hrsg.): Sitzungsberichte der Gesellschaft Naturforschender Freunde zu Berlin. Keltern: Goecke \& Evers, Bd. 52, S. $55-87$.

Sperling, Karl (2020): „Das Humangenomprojekt und die Evolution des Menschen“. In: Walter Sudhaus/Andreas Wessel (Hrsg.): Sitzungsberichte der Gesellschaft Naturforschender Freunde zu Berlin. Keltern: Goecke \& Evers, Bd. 54, S. 67-88.

Spielmann, Malte/Brancati, Francesco/Krawitz, Peter M. et al. (2012): „Homeotic Arm-to-Leg Transformation Associated with Genomic Rearrangements at the PITX1 Locus“. In: The American Journal of Human Genetics 91(4), S. 629-635.

Spielmann, Malte/Lupiáñez, Darío G./Mundlos, Stefan (2018): „Structural Variation in the 3D Genome“. In: Nature Reviews Genetics 19(7), S. 453-467.

Stearns, S.C./Koella, J.C. (2008): Evolution in Health and Disease. Oxford: Oxford University Press.

Stearns, Stephen C. (2017): „Outstanding Research Opportunities at the Interface of Evolution and Medicine“. In: Nature Ecology \& Evolution 2(1), S. 3-4.

Stearns, Stephen C./Medzhitov, Ruslan (2015): Evolutionary Medicine. Sunderland, MA: Sinauer Associates.

The Lancet (2017a): „Climate Change and Health“. http://www.thelancet.com/infographics/ climate-and-health, besucht am 29.6.2020.

The Lancet (2017b): „What Can Evolutionary Theory Do for Public Health?“. In: The Lancet 390(10093), S. 430.

Trevathan, Wenda R./Smith, E. O./McKenna, James J. (Hrsg.) (1999): Evolutionary Medicine. Oxford: Oxford University Press. 
Uncommon Descent (2018): „,,Core Principles of Evolutionary Medicine“ Still Clinically Useless“. https://uncommondescent.com/intelligent-design/core-principles-of-evolutionarymedicine-still-clinically-useless/, besucht am 24.9.2020.

Veltman, Joris A./Brunner, Han G. (2012): „De novo Mutations in Human Genetic Disease“. In: Nature Reviews Genetics 13(8), S. 565-575.

Virchow, Rudolf (1858): Die Cellularpathologie in ihrer Begründung auf physiologische und pathologische Gewebelehre. Berlin: A. Hirschwald.

Vollmer, Gerhard (2016): Im Lichte der Evolution: Darwin in Wissenschaft und Philosophie. Leipzig: Hirzel.

Warren, Matthew (2018): „Mum's a Neanderthal, Dad's a Denisovan: First Discovery of an Ancient-human Hybrid“. In: Nature 560(7719), S. 417-418.

Weismann, August (1885): Die Continuitat des Keimplasma's als Grundlage einer Theorie der Vererbung. Jena \& Naumburg an der Saale: Fischer Verlag.

Williams, Alison L./Grogan, Sarah/Clark-Carter, David et al. (2013): „Appearance-based Interventions to Reduce Ultraviolet Exposure and/or Increase Sun Protection Intentions and Behaviours: A Systematic Review and Meta-analyses“. In: British Journal of Health Psychology 18(1), S. $182-217$.

Williams, George C. (1957): „Pleiotropy, Natural Selection, and the Evolution of Senescence“. In: Evolution 11(4), S. 398. 